

\title{
Fatores de risco e de proteção na adaptação à aposentadoria
}

\author{
Risk and protection factors in retirement adaptation
}

Cristineide Leandro-França ${ }^{[0]}$, Sheila Giardini Murta ${ }^{[b]}$

\footnotetext{
${ }^{\text {[a] }}$ Psicóloga da Universidade de Brasília, Mestre e Doutoranda em Psicologia Clínica e Cultura pela Universidade de Brasília, End.Departamento de Psicologia Clínica e Cultura, Instituto de Psicologia, Universidade de Brasília, Campus Darcy Ribeiro, Brasília, DF, CEP 70910-900. E-mail: cristineide@unb.br

${ }^{[b]}$ Profa. Adjunta no Departamento de Psicologia Clínica da Universidade de Brasília - UnB. End. Departamento de Psicologia Clínica, Instituto de Psicologia, Universidade de Brasília, Campus Darcy Ribeiro, Brasília, DF, CEP 70910-900.

E-mail:giardini@unb.br
}

Recebido: 15/11/2011 Received: 11/15/2011

Aprovado: 05/06/2012 Approved: 06/05/2012

\section{Resumo}

Este ensaio tem como objetivo descrever fatores de risco e de proteção individuais, psicossociais e organizacionais que dificultam ou facilitam a adaptação do indivíduo à aposentadoria, a partir da literatura especializada. Destacam-se como fatores de risco as condições adversas: poucos recursos financeiros, doenças, ausência de rede social e laços familiares, falta de planejamento e aposentadoria involuntária. Atuam como fatores protetivos na adaptação à aposentadoria: ter autoeficácia, maior renda, educação, saúde, lazer, praticar atividades físicas, ser casado, ter rede social e realizar trabalho voluntário. São discutidas implicações destes achados para o planejamento de programas preventivos, os quais devem ser focados na ampliação de recursos pessoais, familiares e ambientais que facilitem o enfrentamento à transição para a aposentadoria.

Palavras-chave: Aposentadoria. Comportamento. Planejamento.

\section{Abstract}

This paper aims to describe individual, psychosocial and organizational risk and protection factors that hinder or facilitate the individual's adaptation to retirement. Stand out as risk factors for the adverse conditions: limited financial resouces, diseases, lack of social network and family ties, lack of planning and involuntary retirement. As protective factors in the adjustment to retirement there are: self-efficacy, high income, education, health, leisure, physical activities, being married, having a social network and perform volunteer work. Implications of these findings for planning prevention programs, which should be focused on the expansion of personal, family and environmental resources that facilitate facing the transition to retirement are discussed.

Keywords: Retirement. Behavior. Planning.

Psicol. Argum. 2014 jan./mar., 32(76), 33-43 


\section{Introdução}

A passagem para a aposentadoria, como outras transições no trabalho, pode gerar tensão e dificuldades na adaptação do indivíduo a um novo estágio da vida (Fouad \& Bynner, 2008). Embora as circunstâncias dessa etapa sejam enfrentadas de forma saudável por alguns trabalhadores, outros são afetados em sua saúde física e psicológica. Mudanças na qualidade de vida decorrentes da aposentadoria tornam-se ainda mais relevantes com o aumento da expectativa de vida mundial. No Brasil, a população está envelhecendo alcançando uma expectativa de vida de 73 anos. Em 2050 estima-se que a esperança de vida será de 81 anos e que o Brasil terá o mesmo número de pessoas idosas e jovens, representadas por $18 \%$ da população geral ou 47 milhões de pessoas nessas faixas etárias (França \& Soares, 2009). Considerando esse aumento na longevidade, são relevantes estudos acerca das dimensões positivas e negativas que afetam a vida dos trabalhadores durante o processo da aposentadoria. Isto pode facilitar a tarefa de formuladores de políticas públicas, gestores, profissionais e pesquisadores no planejamento de medidas de proteção à saúde das pessoas na fase tardia da vida.

A situação de estresse desencadeada pela transição para a aposentadoria vem chamando atenção dos órgãos públicos e sociais brasileiros (Duarte \& Melo-Silva, 2009) que, por meio de estratégias como a Política Nacional do Idoso (Lei 8.842/1994) e o Estatuto do Idoso (Lei 10/741/2003), buscam promover saúde, autonomia, redes de suporte social e melhor condição de vida das pessoas nessa faixa etária. Ainda no contexto da aposentadoria, o governo brasileiro estabeleceu, em 2010, políticas de atenção à saúde do servidor público federal, por meio do Subsistema Integrado de Atenção à Saúde do Servidor - SIASS (MPOG, 2010). Uma das diretrizes dessa política é incentivar o estabelecimento de programas de preparação para a aposentadoria nas instituições públicas federais.

No Brasil, tem sido crescente o interesse por programas de preparação para esta transição de vida (França, 2002; França \& Carneiro, 2009; França \& Soares, 2009; Murta, Caixeta, Souza \& Ribeiro, 2008; Zanelli, Silva \& Soares, 2010; Soares \& Costa, 2011). Contudo, são ainda recentes os estudos nacionais que investigam o impacto da aposentadoria na vida do indivíduo e os fatores relevantes para uma aposentadoria bem-sucedida (Duarte \& Melo-Silva, 2009; Oliveira, Torres \& Albuquerque, 2009). Para aprofundar o conhecimento de tais variáveis, são necessários estudos longitudinais que acompanhem os indivíduos no período pré e pós-aposentadoria.

Entretanto, são escassos na literatura nacional estudos que utilizam métodos longitudinais de investigação. Uma exceção é o estudo qualitativo de Duarte e Melo-Silva (2009), de caráter exploratório, com delineamento longitudinal, que acompanhou 30 trabalhadores, 15 homens e 15 mulheres, com idade de 40 a 69 anos em situação de pré e pós-aposentadoria. Tal estudo tinha como objetivo investigar as expectativas desses trabalhadores, 1 a 3 meses antes da aposentadoria e 12 meses após. Constatou-se que antes de se aposentar existia uma visão estigmatizada com o fato de não trabalhar, que era associada ao envelhecimento, à ociosidade, ao fim da trajetória profissional e à perda da capacidade funcional. Os participantes relataram sentimentos de tensão, ansiedade, medo do novo, do desconhecido, acentuado pela insegurança com relação à instabilidade financeira e à ameaça da perda de sua identidade de trabalhador. Eles também apresentaram expectativas positivas quanto à qualidade de vida, tais como aproveitar o tempo com viagens, lazer e atividades voluntárias. Com a aposentadoria já instituída, os participantes mencionaram sentimento de injustiça pelo baixo valor dos proventos recebidos, mas também a conquista da liberdade, autonomia, cidadania, respeito pessoal e desejo de fortalecer os vínculos familiares.

Em uma revisão de estudos sobre a adaptação à aposentadoria, Wang, Henkens e van Solinge (2011) afirmam que o ajustamento a esta nova fase é um processo contínuo e que pode flutuar dependendo dos recursos que cada um possui e das mudanças destes recursos no decorrer da vida. Nesta revisão, constatou-se que perdas em saúde física, dependência financeira dos filhos, viuvez durante a transição, aposentadoria precoce e por invalidez eram preditores de problemas na adaptação à aposentadoria. As condições favoráveis ao ajustamento foram: planejamento prévio à aposentadoria, saúde física e mental, aposentadoria voluntária, recursos financeiros, lazer, trabalho formal ou voluntário e qualidade da relação conjugal.

Achados como os de Wang et al. (2011) e de outros estudiosos do assunto permitem a identificação 
de condições facilitadoras e dificultadoras de adaptação à aposentadoria e, por consequência, apontam implicações para o planejamento de programas de preparação para esta nova etapa de vida. Apoiando-se nestes pressupostos, o presente ensaio tem como finalidade descrever fatores de risco e de proteção no planejamento para a aposentadoria, a partir da literatura especializada, abordando características individuais, psicossociais e organizacionais que facilitam ou dificultam o ajustamento do indivíduo a esta nova fase da vida. Primeiro, serão conceituados e descritos os fatores de risco e de proteção. Em seguida, serão abordados tais fatores individuais, psicossociais e organizacionais que influenciam a qualidade do ajustamento à aposentadoria para, por fim, ser apresentadas controvérsias da literatura sobre o assunto, indicando as implicações dos resultados no planejamento da aposentadoria.

\section{Fatores de risco na adaptação à aposentadoria}

Entende-se por fatores de risco as condições adversas pessoais ou ambientais que potencializam as vulnerabilidades e aumentam a probabilidade de desfechos negativos no processo de desenvolvimento ou de saúde (Rutter, 1987; Tavares, 2004). Ao pesquisar a relação entre aposentadoria e fatores de risco para o ajustamento à aposentadoria, Canizares (2009) agrupou os preditores de risco em três fatores: psicológicos, sociais e de estilo de vida. 0 primeiro está relacionado à perda do papel regulador do emprego; o segundo, às condições socioeconômicas em que a aposentadoria ocorre; e o terceiro, à possibilidade de comprometimento da capacidade funcional decorrente de hábitos inadequados nos anos que antecedem a aposentadoria. Uma síntese dos fatores de risco encontrados na literatura especializada encontra-se disponível na Tabela 1.

Somam-se a esses estressores relativos à aposentadoria outros comuns nesta etapa do desenvolvimento, como a morte dos progenitores, a saída dos filhos de casa e o declínio biológico (Murta et al., 2008). Pode-se prever que estes eventos adversos de vida, sobretudo em coocorrência, com alta intensidade e longa duração, associados à escassez de recursos de enfrentamento, estejam relacionados aos danos à saúde mental dos idosos, como é o caso do suicídio. No Brasil, em 2001, $13,84 \%$ das mortes por suicídios ocorreram em idosos, principalmente na região Sul. Em 2004, as taxas de suicídio aumentaram em pessoas com idade de 80 anos ou mais, também na região Sul - 21,46\% (Oliveira \& Botega, 2006). 0 isolamento social, sentimentos de solidão e inutilidade,

Tabela 1 - Fatores de risco no planejamento da aposentadoria

\begin{tabular}{lll}
\hline Característica & Fatores de Risco & Autor, ano \\
\hline Pessoal & Declínio da saúde física e problemas de saúde & Quick \& Moen, 1998; Kim \& Moen, 2002; França, \\
& pessoal e familiar & 2002; van Solinge \& Henkens, 2008; Murta et al., 2008; \\
& & Henkens e van Solinge, 2011 \\
& Escassez e estresse financeiro & Gallo, Bradley, Siegel, \& Kasl, 2000; França, 2002; Price \\
& & \& Joo, 2005; Pinquart \& Schindler, 2007; Canizares, \\
& Hábitos não saudáveis anos antes da aposentadoria & Canizares, 2009 \\
Psicossocial & Sofrimento psíquico, depressão, ansiedade & Fletcher \& Hansson, 1991; Floyd et al., 1992; Bos- \\
& & se, Aldwin, Levenson, \& Spiro, 1993; van Solinge \& \\
& & Henkens, 2008 \\
& Dificuldade no convívio marital & França, 2002 \\
& Perda do papel regulador do trabalho & Floyd et al., 1992; Moen, 1996; Quick \& Moen, 1998; \\
& & França, 2002; Canizares, 2009 \\
Aposentadoria Involuntária & Fouad \& Bynner, 2008; De Vries, 1979; Wong \& Earl, \\
& Aposentadoria Abrupta & 2009; Pinquart \& Schindler, 2007 \\
& Desemprego antes da aposentadoria & Quick \& Moen, 1998; Wang, Henkens \& van Solinge,
\end{tabular}

Psicol. Argum. 2014 jan./mar., 32(76), 33-43 
atitude hostil da sociedade para com o idoso, perda de prestígio e a depressão estão entre os fatores de risco para a ocorrência de suicídio nesta faixa etária (Corrêa \& Barrero, 2006). A aposentadoria, quando não planejada, pode intensificar tais sentimentos de solidão e inutilidade, em virtude da perda da ocupação, da rotina, dos vínculos e, não raro, da diminuição de ganhos financeiros. Entretanto, sabe-se que esse não é um cenário uniforme, uma vez que há também experiências de aumento de satisfação com a vida após a aposentadoria (Wang et al., 2011).

\section{Fatores de proteção na adaptação à aposentadoria}

Fatores de proteção consistem em recursos que promovem a resiliência, melhoram ou alteram os repertórios pessoais a determinados riscos de desadaptação (Rutter, 1987). Entre os fatores de proteção fundamentais para o desenvolvimento estão: (a) atributos pessoais (autonomia, autoestima, autoeficácia, bem-estar subjetivo e competência emocional); (b) rede de apoio social positiva (recursos institucionais, relações com amigos, comunidades e colegas de trabalho) e (c) coesão familiar (Rutter, 1987). Especificamente na aposentadoria, podem atuar como fatores protetivos aqueles identificados por Wang et al. (2011): planejar a aposentadoria antecipadamente, ter saúde, aposentar-se voluntariamente, ter autonomia financeira, lazer, ter ocupação e vivenciar boa relação com o cônjuge. Estes e outros fatores protetivos identificados na literatura estão sumarizados na Tabela 2 .

0 suporte das pessoas e redes sociais é significativo para a qualidade e adaptação às novas demandas. Os recursos sociais funcionam como fatores de proteção e tornam o indivíduo capaz de enfrentar vulnerabilidades (Tavares, 2004). Considerando os recursos protetivos sociais, a Organização Mundial da Saúde - OMS recomenda políticas e ações que garantam mobilidade, independência e saúde física

Tabela 2 - Fatores de proteção no planejamento da aposentadoria

\begin{tabular}{|c|c|c|}
\hline Característica & Fatores de Proteção & Autor, ano \\
\hline \multirow[t]{6}{*}{ Pessoal } & Maior escolaridade & Petkoska \& Earl, 2009 \\
\hline & Relacionamento marital & $\begin{array}{l}\text { Mutran, Reitzes \& Fernandez, 1997; França, 2002; Pinquart \& } \\
\text { Schindler, 2007; Wang et al., } 2011\end{array}$ \\
\hline & Carreira contínua & Quick \& Moen, 1998 \\
\hline & $\begin{array}{l}\text { Status socioeconômico e segurança } \\
\text { financeira }\end{array}$ & $\begin{array}{l}\text { Gallo, Bradley, Siegel, \& Kasl, 2000; Price \& Joo, 2005; Pinquart \& } \\
\text { Schindler, } 2007\end{array}$ \\
\hline & $\begin{array}{l}\text { Saúde física e psicológica e planejamen- } \\
\text { to em saúde }\end{array}$ & $\begin{array}{l}\text { Richardson \& Kilty, 1991; Mutran et al., 1997; Quick \& Moen, 1998; } \\
\text { Breslow, Rúben, \& Wallace, 2000; van Solinge \& Henkens, 2008; } \\
\text { Donaldson, Earl \& Muratore, 2010; Wang et al., } 2011\end{array}$ \\
\hline & $\begin{array}{l}\text { Mudança no estilo de vida Atividade } \\
\text { física e de lazer antes da aposentadoria }\end{array}$ & $\begin{array}{l}\text { Kim \& Feldman, 2000; Iso-Ahola, Jackson, \& Dunn, 1994; Kim \& } \\
\text { Feldman, 2000; França \& Vaughan, 2004; Oliveira et al., 2009; }\end{array}$ \\
\hline \multirow[t]{4}{*}{ Psicossocial } & $\begin{array}{l}\text { Autoeficácia, senso de domínio, locus de } \\
\text { controle }\end{array}$ & $\begin{array}{l}\text { Kim \& Moen, 2002; Duarte \& Melo-Silva, 2009; Muratore \& Earl, } \\
2010\end{array}$ \\
\hline & Rede social positiva, laços familiares & $\begin{array}{l}\text { Tavares, 2004; França \& Vaughan, 2004; Greller \& Richtermeyer, } \\
\text { 2006; Wang, Zhan, Liu, \& Shultz, 2008; França \& Carneiro, 2009; } \\
\text { Oliveira et al., 2009, Duarte \& Melo-Silva, } 2009\end{array}$ \\
\hline & Atividade voluntária & van Solinge \& Henkens, 2008; Duarte \& Melo-Silva, 2009 \\
\hline & Planejamento para a aposentadoria & $\begin{array}{l}\text { Fletcher \& Hansson,1991; Feldman, 1994; Moen,1996; Mutran et } \\
\text { al., 1997; França, 2002; WHO, 2002; Denton et al., 2004; Rodrigues, } \\
\text { Ayabe, Lunardelli, \& Canêo, 2005; Wang, Zhan, Liu, \& Shultz, 2008; } \\
\text { Mutran et al., 2008; França \& Soares, 2009; França \& Carneiro, } \\
\text { 2009; Lawrence \& Roush, 2010; Zanelli, Silva, \& Soares, 2010; } \\
\text { Wang et al., 2011; }\end{array}$ \\
\hline \multirow[t]{2}{*}{ Organizacional } & Aposentadoria voluntária & Wang et al., 2011 \\
\hline & Aposentadoria gradual & De Vries, 1979; Wong \& Earl, 2009 \\
\hline
\end{tabular}


e psicológica dos trabalhadores e aposentados à medida que envelhecem (WHO, 2002). Entre essas medidas estão a flexibilização de horários, necessidade de capacitação dos trabalhadores mais velhos, redução do preconceito quanto à idade, harmonia nas equipes intergeracionais e a promoção de programas de preparação para aposentadoria aos que desejam se aposentar.

Considerando o panorama descrito, ao se examinar a literatura nacional e internacional, percebe-se que a investigação do tema adaptação à aposentadoria tem sido feita tomando-se como unidade de análise características individuais, psicossociais e organizacionais que afetam este processo, conforme estão abordadas a seguir.

\section{Características individuais na adaptação à aposentadoria}

Características sociodemográficas como gênero, estado civil, educação, estilo de vida e aspectos financeiros podem atuar como risco ou proteção no processo da aposentadoria. Em relação ao gênero, existem contradições quanto à sua influência numa aposentadoria promissora. Os estudos mais antigos (Hatch, 1987; Atchley, 1989) evidenciam que mulheres possuem atitudes significativamente mais positivas que os homens acerca da aposentadoria. No entanto, estudos mais recentes (Quick \& Moen, 1998; Kim \& Moen, 2002; França, 2002; Price \& Joo, 2005) dividem-se quanto a essas hipóteses. França (2002) hipotetiza que, em geral, a mulher parece se adaptar melhor à aposentadoria, pois consegue dividir seus papéis como esposa, avó, mãe e filha na sociedade. 0 fato de a mulher se engajar em outras atividades após o afastamento do trabalho pode ser um fator protetivo e preditor de adaptação. Entretanto, Floyd et al. (1992) sugere que mulheres apresentam mais atitudes negativas com relação à aposentadoria, constatando em estudos com aposentados que elas apresentavam menos satisfação na aposentadoria e experienciavam mais estresse em seu início.

Os fatores que contribuem para a qualidade de vida na aposentadoria foram investigados por Quick e Moen (1998). Estes autores relataram, embora a diferença seja pequena, que homens experimentam mais satisfação que as mulheres nesse período. 0 aumento da qualidade de vida na aposentadoria, para elas, está associado à boa saúde, uma carreira contínua (menos lacunas na sua história de empregos), uma aposentadoria antecipada (embora não antes do previsto) e uma boa renda na aposentadoria. Os fatores-chave na adaptação à aposentadoria para os homens foram: ter uma boa saúde, planejamento substancial na pré-aposentadoria e ter se aposentado por razões motivadas internamente, tais como poder realizar atividades prazerosas e de lazer. Ainda como resultados dessa pesquisa, ter uma renda suficiente e não precisar mais trabalhar por muito tempo, ou então receber incentivos financeiros oferecidos pelo empregador, tornam a aposentadoria uma opção atrativa e satisfatória para os homens. No entanto, aposentar por forças externas como demissão, problemas de saúde pessoal e familiar estão associados negativamente à qualidade na aposentadoria, principalmente para os homens.

A influência dos fatores demográficos como idade, gênero, educação e variáveis psicológicas na adaptação para a aposentadoria foram avaliadas numa instituição financeira por Petkoska e Earl (2009). Com relação ao gênero, os resultados associam as mulheres, em detrimento dos homens, a um melhor planejamento da aposentadoria quanto ao domínio interpessoal, da saúde e do lazer. Uma razão para as mulheres estarem mais engajadas em comportamento saudável positivo é seu consumo ativo de informações sobre saúde e serviços de cuidados com a saúde. Ainda nessa pesquisa, a educação foi positivamente relacionada ao planejamento em saúde para a aposentadoria. Os autores salientam que os participantes com maior escolaridade possuem mais informações, conhecimento e acesso sobre questões relativas a esse âmbito. 0 bom nível de escolaridade é um fator de proteção que possibilita aos indivíduos qualificados uma preparação para o pós-carreira. Sendo assim, políticas de incentivo à educação ao longo da vida são recomendadas como facilitadoras da adaptação à aposentadoria (França \& Soares, 2009).

Uma descoberta relevante aparece nos estudos sobre estado civil. 0 fato de estar casado pode ser um preditor de uma aposentadoria bem-sucedida. Comparados a pessoas solteiras, os adultos casados têm relatado maior número de atitudes positivas com relação à aposentadoria e apresentado níveis mais altos de satisfação nesse período (Mutran, Reitzes, \& Fernandez, 1997). Condições que promovem tal comportamento não foram exploradas

Psicol. Argum. 2014 jan./mar., 32(76), 33-43 
nesses estudos. Como hipótese, pode-se prever que a qualidade da relação conjugal promove emoções positivas, permite a troca de apoio social, favorece a construção de projetos de vida compartilhados e a adoção de um estilo de vida saudável quanto à alimentação, sono e atividade física.

Estilo de vida é outro fator a ser considerado na aposentadoria. Melhor saúde física e psicológica tem sido associadas a uma aposentadoria mais satisfatória (Richardson \& Kilty, 1991; Mutran et al., 1997; Quick \& Moen, 1998; van Solinge \& Henkens, 2008; Donaldson et al., 2010). A saúde do indivíduo na aposentadoria normalmente é influenciada tanto por práticas de saúde atuais como anteriores à aposentadoria, tais como a prática regular de atividade física, a moderação no consumo de álcool e o abandono do fumo (Breslow, Rúben, \& Wallace, 2000). Assim, para uma aposentadoria satisfatória, é vital que as pessoas se engajem em práticas de promoção da saúde e tenham planos individuais com relação à saúde com antecedência à sua aposentadoria.

As atividades de lazer também têm sido consideradas importantes para satisfação na aposentadoria. Elas servem como fonte de contato social e influenciam positivamente na qualidade de vida dos aposentados (Oliveira et al., 2009). Iso-Ahola, Jackson e Dunn (citados por Petroska \& Earl, 2009) apresentam evidências de que indivíduos que se envolvem com atividades de lazer antes da aposentadoria apresentam uma tendência a continuar a praticá-las na aposentadoria. Salienta-se que a prática de lazer pode ser facilitada por condições de saúde e segurança financeira, e este conjunto de fatores, por sua vez, afeta a probabilidade do indivíduo se engajar em atividades sociais (Kim \& Feldman, 2000). Nota-se, portanto, uma interrelação entre os diversos fatores protetivos, que podem se influenciar reciprocamente.

O status socieconômico e a segurança financeira aparecem como fatores de risco (quando insuficientes para gerar autonomia) ou preditores de sucesso na aposentadoria (quando suficientemente presentes). Uma renda maior promove uma aposentadoria mais satisfatória, enquanto uma renda inadequada e estresse financeiro estão associados à insatisfação e uma experiência negativa com esse período (Gallo et al., 2000; Price \& Joo, 2005; Noone, Stephens, \& Alpass, 2010).
Embora trabalhadores que possuem altos cargos de prestígio e alta renda percam benefícios ao se aposentar, eles possuem mais recursos para se adaptarem à aposentadoria (Pinquart \& Schindler, 2007), pois a alta renda permite que usufruam de maior número de oportunidades sociais e de lazer. Neste sentido, manter o alto padrão financeiro na aposentadoria é algo que inquieta os executivos brasileiros. Ao analisar as atitudes de ganhos e perdas esperadas na aposentadoria, 517 executivos residentes no Brasil e na Nova Zelândia, França e Vaughan (2008) apontaram que a maioria dos executivos de grandes organizações brasileiras se preocupam com a posição financeira na aposentadoria mais que os neozelandeses.

Para muitos trabalhadores a aposentadoria pode ocasionar perda salarial, impacto no orçamento familiar e, consequentemente, na qualidade de vida (Duarte \& Melo-Silva, 2009). Os gastos com manutenção da saúde, que podem aumentar com a idade, é uma realidade enfrentada pela população brasileira que sofre nos últimos anos com políticas governamentais de redução dos benefícios previdenciários.

\section{Características psicossociais na adaptação à aposentadoria}

Características psicossociais referem-se à influência dos fatores psicológicos, sociais e do planejamento na aposentadoria. Entre os fatores psicológicos estão os sentimentos como autoeficácia, senso de domínio e características emocionais de quadros clínicos como a depressão.

De acordo com os estudos sobre os fatores psicológicos, senso de domínio, autonomia, autoeficácia e locus de controle (acreditar que as ações pessoais influenciam nos resultados positivos e negativos da vida), são recursos que podem ser utilizados por pessoas em transição para aposentadoria (Kim \& Moen, 2002; Muratore \& Earl, 2010). Esses recursos funcionam como mecanismos de proteção e estão relacionados à capacidade que os indivíduos têm de controlar os objetivos da sua vida.

Evidências preliminares sugerem que altos níveis de domínio e autoeficácia podem ter um impacto positivo significativo sobre bem-estar na aposentadoria. Em estudos com homens e mulheres, Kim e Moen (2002) constataram que, durante a transição para aposentadoria, os indivíduos que têm domínio, 
autonomia ou controle sobre suas vidas apresentam menor número de sintomas depressivos. Para muitos indivíduos o afastamento do trabalho pode acarretar sentimentos de solidão e episódios depressivos, principalmente para aqueles que têm no trabalho uma fonte significativa de interação social. Por isso, o planejamento interpessoal para aposentadoria desempenha um papel importante na garantia de uma aposentadoria satisfatória (Bosse, Aldwin, Levenson, \& Spiro, 1993).

Dentre os fatores sociais, família e amigos aparecem como recursos de proteção nessa fase de transição. Relacionamento satisfatório com a família e com os amigos são recursos importantes de apoio, fonte de identidade e de prazer na aposentadoria (França \& Vaughan, 2004; Greller, \& Richtermeyer, 2006; Wang, Zhan, Liu \& Shultz, 2008; França \& Carneiro, 2009). No contexto social, destacam-se ainda os achados de van Solinge e Henkens (2008) sobre o papel do trabalho voluntário. Esses autores se colocam como os primeiros a mostrar o efeito benéfico causado pelo engajamento em trabalhos voluntários na pré-aposentadoria. A atividade voluntária está mundialmente em ascenção na população de meia-idade e mais velha (Duarte \& Melo-Silva, 2009). 0 voluntariado pode proporcionar estabilidade e suporte na transição para a aposentadoria e funciona como estratégia de ocupação, de distração, um recurso relevante no estilo de vida nessa fase. Além disso, pessoas que realizam atividades voluntárias relatam vivenciar sensações de prazer e bem -estar decorrentes dessa prática.

0 planejamento para aposentadoria também tem sido apontado como fator de proteção na adaptação pós-aposentadoria. Alguns estudos apontam que o planejamento da aposentadoria promove atitudes positivas e bem-sucedidas nos períodos antes e durante a aposentadoria (Mutran et al., 1997; Wang et al., 2008), diminui os níveis de ansiedade (Fletcher \& Hansson, 1991) e aumenta a satisfação se comparado aos que não planejaram (Feldman, 1994; Moen, 1996). Programas multimodais que incluem diversos grupos de fatores protetivos, os individuais, psicossociais e organizacionais, possivelmente tendem a ser mais efetivos (França \& Carneiro, 2009; Lawrence \& Roush, 2010), em comparação àqueles restritos ao planejamento financeiro (Petkoska \& Earl, 2009). Adicionalmente, tais programas devem favorecer um planejamento contínuo para garantir o bem-estar nessa fase da vida
(Denton et al., 2004). Assim, planejamentos abrangentes e multitemáticos anteriores à aposentadoria podem ser preditores importantes de uma aposentadoria mais satisfatória.

\section{Características organizacionais na adaptação à aposentadoria}

Nesse campo são exploradas decisões organizacionais que influenciam na transição para a aposentadoria, condições de saída da força de trabalho e como essas condições podem alterar a adaptação à aposentadoria de um indivíduo. Ao considerar o significado do trabalho e as consequências que sua perda pode causar na vida de uma pessoa, é importante analisar as razões que remetem à decisão de aposentar-se. Essas condições podem ser voluntárias (por vontade própria, tempo de contribuição ou idade compatível) ou involuntárias (por invalidez ou compulsória). Na aposentadoria voluntária o indivíduo deseja se aposentar, enquanto na involuntária a pessoa é compelida por uma situação que não estava preparada para aceitar.

Ao desenvolver um instrumento para avaliar a satisfação e a percepção das experiências relacionadas à aposentadoria, Floyd et al. (1992) descobriram diferenças entre as pessoas que se aposentavam involuntariamente e voluntariamente. Indivíduos que se aposentaram involuntariamente obtiveram um escore mais alto no fator "pressão exercida pelo empregador". A influência dessa variável torna mais difícil o período de transição, gera menos satisfação e prazer com a aposentadoria e ocasiona dificuldades no engajamento às atividades física, social e de lazer, após a aposentadoria, em relação às pessoas que se aposentaram de forma voluntária. Em contrapartida, indivíduos que se aposentaram voluntariamente manifestaram um desejo relativamente alto de perseguir seus próprios interesses. Isto facilitou a transição e resultou no aumento da satisfação, maior número de fontes de prazer e uma adaptação positiva à aposentadoria.

A forma como a aposentadoria acontece, seja abrupta ou gradual, interfere na maneira como o indivíduo se ajusta à nova realidade. 0 choque inicial, vivenciado por alguns indivíduos, pode ser amenizado se a aposentadoria ocorre de forma progressiva, mais lentamente (De Vries, 1979). A forma gradual proporciona mais tempo para a adaptação às

Psicol. Argum. 2014 jan./mar., 32(76), 33-43 
mudanças nos papéis e estilo de vida que uma saída abrupta. Planejar a aposentadoria é um dos passos para facilitar essa decisão e reduzir a ansiedade associada a esse evento (Wong \& Earl, 2009). Embora o afastamento gradual seja o mais indicado, nem sempre isso é possível, principalmente quando se trata de decisões organizacionais, como demissões, reestruturação, redução de custos ou oferecimento de incentivos para uma aposentadoria antecipada.

Os Programas de Preparação para Aposentadoria, oferecidos pelas organizações, podem facilitar o planejamento gradual e a prevenção às dificuldades na adaptação (Soares \& Costa, 2011). Estas ações oportunizam a reflexão sobre pontos favoráveis e desfavoráveis na passagem para a aposentadoria, além da busca de alternativas de enfrentamento, conforme proposta dos programas referenciados na literatura nacional (Soares, Costa, Rosa \& Oliveira, 2007; Costa \& Soares, 2009; França \& Soares, 2009; França \& Carneiro, 2009; Soares \& Costa, 2011).

Segundo Wang et al. (2011), existem muitos programas de planejamento para aposentadoria que são oferecidos por governos, organizações públicas e privadas, assim como muitas publicações de autoajuda sobre o tema. Entretanto, são necessárias mais pesquisas que avaliem estes programas e comparem os diversos tipos de intervenções, com foco na avaliação longitudinal de resultados, identificação de formatos mais ou menos efetivos e construção de programas para trabalhadores com características demográficas distintas.

A participação de psicólogos nestas ações também merece atenção, seja do ponto de vista da pesquisa ou da formação profissional. A literatura aponta a relevância da colaboração desses profissionais na elaboração de programas pautados em variáveis que contemplem a qualidade de vida laboral (Rodrigues et al., 2005) e no auxílio aos indivíduos que enfrentam o afastamento do trabalho de forma voluntária ou involuntária, gradual ou abrupta (Fouad \& Bynner, 2008). Para estes autores, psicólogos organizacionais e do trabalho podem contribuir para a criação de políticas de gestão de pessoas que favoreçam a transição voluntária e gradual dos trabalhadores e no reforço de recursos existentes. Além disso, psicólogos clínicos e da saúde podem auxiliar os trabalhadores que atravessam transições involuntárias e abruptas a desenvolver estratégias de enfrentamento para prevenção ou minimização de danos à saúde, como a depressão e o estresse.

\section{Considerações finais}

A capacidade do indivíduo ajustar-se às mudanças decorrentes da aposentadoria difere para cada pessoa, conforme seus recursos (Wang et al., 2011). Para que esta trajetória seja satisfatória e bem-sucedida, deve-se considerar os fatores de risco e de proteção que interferem nessa transição. A literatura clássica e atual analisa a preparação e a adaptação à aposentadoria considerando características pessoais, psicossociais e organizacionais que afetam estes processos. 0 presente ensaio seguiu essas unidades de análise ao discutir fatores de risco e de proteção e a influência dessas características no planejamento e adaptação à aposentadoria.

Um aspecto importante, referido na literatura como um preditor positivo para o enfrentamento das situações difíceis nessa fase de transição, é a capacidade humana de controlar e dominar a sua própria vida e seus objetivos. Esta capacidade tem sido associada a locus de controle interno, percepções de autoeficácia e altos níveis de autonomia. É relevante que profissionais que trabalhem com este público fiquem atentos a essas variáveis e as incluam em suas ações, sejam estas preventivas ou assistenciais, individuais ou em grupo, de curto ou longo prazo.

Estudos internacionais, discutidos neste ensaio, destacam as características individuais, psicossociais e organizacionais como risco e proteção no planejamento da aposentadoria. Entretanto, questiona-se a generalização desses resultados à população brasileira, considerando que as regras de aposentadoria, condições políticas, financeiras e sociais diferem das de outros países. São ainda poucos os estudos nacionais voltados para as expectativas de trabalhadores brasileiros frente à aposentadoria (França e Vaughan, 2008) e suas experiências de adaptação pós-aposentadoria (Duarte e Melo-Silva, 2009). São necessários estudos longitudinais com amostras brasileiras, que investiguem as especificidades e as generalidades entre regiões, categorias profissionais e classes sociais.

Apesar das limitações quanto à validade externa, os achados deste ensaio demonstram um panorama dos estudos sobre fatores de risco e proteção no planejamento para aposentadoria e apontam potencialidades encontradas nesse campo do conhecimento. Considerando tais resultados, os programas preventivos na transição para a aposentadoria deveriam focar em ações pessoais, dirigidas a 
mudanças comportamentais, como fortalecimento da autoeficácia, redes sociais, autonomia e laços familiares, além de ações organizacionais, como políticas de aposentadoria gradual e voluntária. Devem ser tomados cuidados no desenho dos programas preventivos, para que sejam multicomponentes em seu conteúdo, abrangendo diferentes fatores protetivos, para além de habilidades de manejo das finanças, conforme proposto por Petkoska e Earl (2009).

Além disso, são necessárias avaliações sistemáticas, contínuas e cuidadosas desses programas (França, 2002; Soares et al., 2007; Murta et al, 2008; Zanelli et al., 2010), utilizando critérios que avaliem processos, resultados e impactos, com o intuito de proporcionar mudanças efetivas para uma aposentadoria bem-sucedida. A análise desses programas pode auxiliar profissionais, pesquisadores, formuladores de políticas e gestores, nas decisões e planejamentos de ações direcionadas à aposentadoria, bem como no investimento e administração adequada dos recursos públicos destinados a este fim. 0 cumprimento desta agenda de pesquisa poderá contribuir na implantação de políticas e ações preventivas que colaborem para a promoção de aposentadorias bem vividas e qualidade de vida deste segmento populacional, cada vez mais crescente.

\section{Referências}

Atchley, R. C. (1989). A continuity theory of normal aging. Gerontologist, 29(2), 183-190.

Breslow, L., Reuben, D., \& Wallace, S. (2000). Introduction to special issue on health promotion among the elderly. American Journal of Health Promotion, 14(6), 341-342.

Bossé, R., Aldwin, C. N., Levenson, R., Spiro, A., III, \& Mroczek, D. K. (1993). Change in social support after retirement: Longitudinal findings from the Normative Aging Study. Journals of Gerontology: Psychological Sciences and Social Sciences, 48(4), 210-217.

Canizares, J.C. (2009). Fatores de risco à senilidade na transição à aposentadoria. (Tese de Doutorado), Universidade de São Paulo, São Paulo.

Corrêa, H., \& Barrero, S. P. (2006). Fatores de risco para o suicídio durante o ciclo da vida humana. In Suicídio: Uma morte evitável. São Paulo: Atheneu.
Costa, A. B., \& Soares, D. H. P. (2009). Orientação psicológica para a aposentadoria. Revista Psicologia: Organizações e Trabalho, 9(2), 97-108.

De Vries, M. F. (1979). Is there life after retirement?. California Management Review, 22, 69-76.

Denton, M. A., Kemp, C. L., French, S., Gafni, A., Joshi, A., \& Rosenthal, C. J. (2004). Reflexive planning for later life. Canadian Journal on Aging, 23, 71-82.

Donaldson, T., Earl, J.K., \& Muratore, A. (2010). Extending the integrated model of retirement adjustment: Incorporating mastery and retirement planning. Journal of Vocational Behavior, 77(2), 279-289.

Duarte, V. C., \& Melo-Silva, L. L. (2009). Expectativas diante da aposentadoria: Um estudo de acompanhamento em momento de transição. Revista Brasileira de Orientação Profissional, 10(1), 45-54.

Feldman, D. (1994). The decision to retire early: A review and conceptualization. Academy of Management Review, 19(2), 285-311.

Fletcher, W. L., \& Hansson, R. O. (1991). Assessing the social components of retirement anxiety. Psychology and Aging, 6(1), 76-85.

Floyd, F., Haynes, S., Doll, E., Winemiller, D., Lemsky, C., Burgy, T., M. Werle, M., \& Heilman, N. (1992). Assessing retirement satisfaction and perceptions of retirement experiences. Psychology and aging, 7(4), 609-621.

Fouad, N., \& Bynner, J. (2008). Work transitions. American Psychological, 63(4), 241-251.

França, L.H. (2002). Repensando a aposentadoria com qualidade: um manual para facilitadores de programas de educação para aposentadoria em comunidades. Rio de Janeiro: CRDE UnATI UERJ.

França, L. H., \& Vaughan, G. (2004). Ganhos e perdas: atitudes dos executivos brasileiros e neozelandeses frente à aposentadoria. Psicologia em Estudo, 13(2), 207-216.

França, L. H., \& Carneiro, V. (2009). Programa de preparação para a aposentadoria: um estudo com

Psicol. Argum. 2014 jan./mar., 32(76), 33-43 
trabalhadores mais velhos em Resende (RJ). Revista Brasileira de Geriatria e Gerontologia, 12(3), 429-447.

França, L. H., \& Soares, D. (2009). Preparação para a aposentadoria como parte da educação ao longo da vida. Psicologia, Ciência e Profissão, 29(4), 738-751.

Gallo, W. T., Bradley, E. H., Siegel, M., \& Kasl, S.V. (2000). Health effects of involuntary job loss among older workers: Findings from the Health and Retirement Survey. Journal of Gerontology: Social Sciences, 55(3), 131-140.

Greller, M. M., \& Ritchtermeyer, S. B. (2006). Changes in social support for professional development and retirement preparation as a function of age. Humans Relations, 59(9), 1213-1234.

Hatch, L. R. (1987). Research on men's and women's retirement attitude. In Borgatta, E. F., \& Montgomory, R. J. V. (Eds). Critical issues in aging policy: Linking research and values (pp.129-160). Califórnia: Sage.

Iso-Ahola, S. E., Jackson, E., \& Dunn, E. (1994). Starting, ceasing, and replacing leisure activities over the life span. Journal of Leisure Research, 26(3), 227-249.

Kim, S., \& Feldman, D. C. (2000). Working in retirement: The antecedents of bridge employment and its consequences for quality of life in retirement. Academy of Management Journal, 43(6), 1195-1210.

Kim, J. E., \& Moen, P. (2002). Retirement transitions, gender and psychological well-being: A life-course, ecological model. Journal of Gerontology, 57(3), 212-222.

Lawrence, S., \& Roush, S. E. (2010). Examining pre-retirement and related services offered to services-users with an intellectual disability in Ireland. Journal of Intellectual Disabilities, 12(3), 239-252.

Ministério do Planejamento, Orçamento e Gestão - MPOG. (2010). Portaria Normativa SRH n. 1261/2010, cap II, art. 2, XV.

Moen, P. (1996). A life course perspective on retirement, gender, and well-being. Journal of Occupational Health Psychology, 1(2), 131-144.
Muratore, A. M., \& Earl, J. K. (2010). Predicting retirement preparation through the design of a new measure. Australian Psychologist, 45(2), 98-111.

Murta, S. G., Caixeta, T. D., Souza, K. L., \& Ribeiro, D. C. (2008). Avaliação de um programa de preparo para a aposentadoria e envelhecimento bem-sucedido. In Murta, S. G. (Org.). Grupos psicoeducativos: Aplicações em múltiplos contextos (pp. 181-196). Goiânia: Porã Cultural.

Mutran, E. J., Reitzes, D. C., \& Fernandez, M. E. (1997). Factors that influence attitudes toward retirement. Research on Aging, 19, 251-273.

Noone, J. H., Stephens, C., \& Alpass, F. (2010). Do men and women differ in their retirement planning? Testing a theoretical model of gendered pathways to retirement preparation. Research on Aging, 32(6), 715-738.

Oliveira, C. F., \& Botega, N. J. (2006). Prevenção do suicídio: Manual dirigido a profissionais das equipes de saúde mental. Ministério da Saúde/SVS, recuperado em 19 de dez. 2010, de http://portal.saude.gov.br/ portal/arquivos/pdf/saude_brasil_2007.pdf

Oliveira, C., Torres, A. R. R., \& Albuquerque, E. S. (2009). Análise do bem estar psicossocial de aposentados de Goiânia. Psicologia em Estudo, 14(4), 749-757.

Petkoska, J., \& Earl, J. K. (2009). Understanding the influence of demographic and psychological variables on retirement planning. Psychology and Aging, 24(1), 245-251.

Pinquart, M., \& Schindler, I. (2007). Changes of life satisfaction in the transition to retirement: A latent-class approach. Psychology and Aging, 22(3), 442-455.

Price, K. P., \& Joo, F. (2005). Exploring the relationship between marital status and women's retirement satisfaction. International Journal of Aging and Human Development, 61, 37-55.

Quick, H. E., \& Moen, P. (1998). Gender, employment and retirement quality: A life course approach to the differential experiences of men and women. Journal of Occupational Health Psychology, 3, 44-64. 
Richardson, V., \& Kilty, K. M. (1991). Adjustment to retirement: Continuity vs. discontinuity. International Journal of Aging and Human Development, 33, 151-169.

Rodrigues, M., Ayabe, N. H., Lunardelli, M. C. F., \& Canêo, L. C. (2005). A preparação para a aposentadoria: 0 papel do psicólogo frente a essa questão. Revista Brasileira de Orientação Profissional, 6, 53-62.

Rutter, M. (1987). Psychosocial resilience and protective mechanisms. American Journal of Orthopsychiatry, 57,316-331

Soares, D. H. P., Costa, A., Rosa, A. M., \& Oliveira, M. L. (2007). Aposenta-ação: Programa de preparação para a aposentadoria. Estudos Interdisciplinares sobre o Envelhecimento, 13, 123-134.

Soares, D.H., \& Costa, A. (2011). Aposent-ação: aposentadoria para a ação. São Paulo: Vetor.

Tavares, M., \& Werlang, B. S. G. (2012). 0 conceito de crise e seus efeitos na prevenção e na intervenção em crise. In Viana, T. C., Diniz, G. S., Costa, L. F., \& Zanello, V. (Orgs.). Psicologia Clínica e Cultura Contemporânea. (pp. 470-493). Brasília: Liber Livro. van Solinge, H., \& Henkens, K. (2008). Adjustment to and satisfaction with retirement: Two of a kind? Psychology and Aging, 23, 422-434.

Wang, M., Zhan, Y. Liu, S., \& Shultz, K. (2008). Antecedents of bridge employment: A longitudinal investigation. Journal of Applied Psychology, 4, 818-830.

Wang, M., Henkens, K., \& van Solinge, H. (2011). Retirement adjustment: a review of theoretical and empirical advancements. American Psychologist, 1, 1-13.

Wong, J., \& Earl, J. (2009). Towards an integrated model of individual, psychosocial, and organizational predictors of retirement adjustment. Journal of Vocational Behavior, 75, 1-13.

World Health Organization -WHO (2002). Active ageing: A policy framework. Recuperado em 8 de dez. 2010, de http://www.who.int/ageing/publications/active/en/index.html

Zanelli, J. C., Silva, N. \& Soares, D. H. (2010). Orientação para aposentadoria nas organizações de trabalho: Construção de projetos para o pós-carreira. Porto Alegre: Artmed.

Psicol. Argum. 2014 jan./mar., 32(76), 33-43 